\title{
Changes in daily loneliness for German residents during the first four weeks of the COVID-I 9 pandemic
}

Susanne Buecker ${ }^{*}$, Kai T. Horstmann ${ }^{2 *}$, Julia Krasko', Sarah Kritzler', Sophia Terwiel', Till Kaiser', and Maike Luhmann'

This is the final accepted version, Nov 18, 2020 (in press, Social Science \& Medicine)

This postprint may differ slightly from the final, copy-edited version of this article.

https://doi.org/10.1016/j.socscimed.2020.11354I

\footnotetext{
'Department of Psychology, Ruhr University Bochum, Germany

${ }^{2}$ Department of Psychology, Humboldt-Universität zu Berlin, Germany
}

\section{Corresponding Author:}

Susanne Buecker, Ruhr University Bochum, Universitätsstraße I50, 4480I Bochum.

Email: Susanne.buecker@rub.de

* equal contributions/shared first authorship 


\begin{abstract}
Rationale: The coronavirus disease 2019 (COVID-19) outbreak has dramatically altered people's social lives due to social restriction measures taken to control the coronavirus spread. Early on, increased loneliness has been publicly discussed as a harmful psychological side effect of these measures. Due to the serious adverse health consequences of loneliness, it is essential to take these concerns seriously and investigate them systematically to allow for evidence-based decision making. Thus far, however, high-resolution empirical evidence of such harmful side effects is rare.
\end{abstract}

Methods: The present preregistered large-scale daily diary study assessed daily loneliness in 4,844 German adults between March 16 and April I2, 2020.

Results and conclusion: Daily loneliness slightly increased during the first two weeks since implementing the pandemic-related measures and slightly decreased thereafter. With increasing age, daily loneliness increased more strongly over the four weeks. Moreover, daily loneliness increased more strongly for parents compared to people without children. Thus, despite some increases in loneliness in some individuals, there was no linear increase in loneliness in response to the first pandemic-related measures in the present sample.

\title{
Keywords
}

loneliness, isolation, Covid-19, daily diary, distancing policies 
The Covid-19 pandemic has dramatically altered people's everyday lives around the globe. Lockdowns, shelter-at-home orders, quarantines, and physical distancing policies forced people to minimize personal encounters. Although these measures have effectively slowed the spread of the coronavirus in most countries (Dehning et al., 2020), they raised concerns about potentially harmful psychological side effects in the general public (Holmes et al., 2020). Early on, media reports warned about a looming loneliness epidemic (Klein, 2020), particularly among those who already have a higher risk of experiencing loneliness (Finley, 2020). Due to the serious adverse health consequences of loneliness (e.g., Holt-Lunstad et al., 2015), it is essential to investigate the development of loneliness systematically to allow for evidence-based decision making.

Why might the Covid-19 pandemic and its related measures affect loneliness? Loneliness is defined as a subjectively perceived lack of meaningful social relationships (Peplau \& Perlman, 1982) that can occur when the actual social relationships or relationship standards change. Due to the pandemic-related measures (e.g., contact restrictions in public), people across different sociodemographic groups may no longer have fulfill their social needs, potentially resulting in increased loneliness. However, one might also consider the pandemic a "prism" that magnifies pre-existing differences in characteristics associated with loneliness. For example, in young adulthood, forming intimate partnerships, managing friendships, and building more extensive social networks are critical developmental tasks (Hutteman et al., 2014). In contrast, older adults tend to selectively narrow their social interactions (English \& Carstensen, 2014). Consequently, for young adults, contact restrictions in public may have been more challenging than for older adults because these restrictions have hindered young adults' natural drive for broad social interaction. However, one may also see the pandemic as an "opportunity" for changes in one's daily social behaviors. For example, the pan- 
demic-related physical distancing policies may have stimulated more digital communication, enabling more social interactions with people who live further away and with whom one would have had less contact without the pandemic.

Hence, the pandemic and its related measures may have had both positive and negative effects on loneliness, and it is unclear which ones prevail at a population level. Consequently, the theoretical basis for predicting a collective loneliness epidemic is not as factual as it may appear. To date, the empirical evidence on this question is inconclusive, with some studies finding increasing loneliness levels since the beginning of the pandemic (Elmer et al., 2020; Entringer et al., 2020; Fujiwara et al., 2020; Sweeny et al., 2020), but others reporting stable loneliness levels (Fancourt \& Steptoe, 2020; Folk et al., 2020; Luchetti et al., 2020).

It is also unclear whether those sociodemographic groups that are ordinarily most at risk for loneliness are also the most vulnerable regarding changes in loneliness during mandated physical distancing. Previous studies on loneliness indicated that loneliness is more prevalent among young adults aged between 18 to 30 years and among old adults aged 80+ years (Hawkley et al., 2020; Luhmann \& Hawkley, 2016), as well as among unemployed people and among people in poorer health conditions (Pinquart \& Sörensen, 2003). A US study found that older adults reported less loneliness overall than younger age groups, but showed an increase in loneliness in the acute phase of the Covid-19 pandemic (Luchetti et al., 2020). The same study also found that people living alone and those with poorer health condition reported higher loneliness at baseline (pre-pandemic; in late January/early February 2020) and did not increase in loneliness during the implementation of physical distancing measures (Luchetti et al., 2020). 
Finally, most studies on psychological side effects of the pandemic-related measures, including those on loneliness, did not use frequent assessments of their outcomes of interest. However, if loneliness levels change in response to the pandemic-related measures, the pattern of change is likely rather complex.Consequently, a high-resolution examination of the daily loneliness trajectories that can identify short-term flare-ups or non-linear changes is necessary but lacking thus far.

We analyzed high-resolution longitudinal data collected daily in a German sample over four weeks (March 16 - April 12, 2020). During these four weeks, the government issued increasingly strict restrictions on social gatherings and physical distancing. Nationwide, the first pandemic-related measures such as school closings and strict regulation of opening hours for restaurants were implemented on March 16, 2020. These measures were intensified on March 22, 2020, including travel bans and contact restrictions in public. On March 30, 2020, the measures were extended beyond the course of this study.

We investigated average changes in daily loneliness (Research Objective 1) and sociodemographic subgroups that are particularly susceptible to increases in daily loneliness (Research Objective 2). Even small increases in daily loneliness, whether at the population level or within specific subgroups, can accumulate over time, may lead to health issues in the future, and can be associated with high economic costs on a society level (Mihalopoulos et al., 2020). Furthermore, understanding who suffers most from physical distancing can inform public policy to allocate resources to those who are particularly at risk of loneliness. 


\section{Materials and Methods}

\section{Recruiting Procedure}

Data were collected in Germany from March 16, 2020, the first day of implementing the nationwide pandemic-related measures, until April 12, 2020 using formr.org (Arslan et al., 2020). Participants were recruited via various online sources (e.g., Facebook, Twitter, Kleiderkreisel, Xing), media reporting (including radio, newspapers/magazines, TV), and personal contacts. After registering for the study and providing informed consent, participants completed a baseline survey. Following the baseline survey, brief daily diary surveys were administered between 5:00 p.m. and 1:00 a.m. on four consecutive days, followed by a three-day break and a weekly review survey. The day after the weekly survey, the next survey cycle started, consisting again of four daily surveys, a break, and a weekly review survey. For a detailed description see the supplementary material (SM; see Open Science Framework).

\section{Sample}

In the present paper, we analyzed data from the first four weeks (i.e., March 16, 2020, to April 12, 2020) of the daily diary study. During these four weeks, $N=4,844$ participants (aged between 18 and 88 years) participated in the study and completed $n=40,016$ daily surveys (75.93\% response rate, $M d n=1,469$ surveys per day). We randomly split the sample into two equally sized subsamples. We used data from Sample 1 to explore the best fitting models to describe changes in daily loneliness over time. We then performed confirmatory tests of these models in Sample 2, thereby obtaining the most robust and replicable estimates of the observed effects (Faraway, 2016; Weston et al., 2019; Yarkoni \& Westfall, 2017). Sample 1 consisted of $N_{1}=2,428$ participants, and Sample 2 of $N_{2}=2,416$ participants. In Sample 1, $n_{1}=20,079$ daily surveys were completed, and in Sample 2, $n_{2}=19,937$ 
daily surveys were completed. For a detailed description of the sample and surveys collected, see SM.

\section{Measures}

In the baseline survey, we assessed the following sociodemographic characteristics: age, gender, years of education, employment status, occupational classification, which we classified into the essential (e.g., health care, public administration) and non-essential sector, relationship status, parental status, and pre-existing health conditions concerning Covid-19. Table S2 presents an overview of all relevant variables assessed at baseline and the distribution of responses.

Daily loneliness was measured using four items ("I felt lonely today", "I felt left out today", "I had no one to turn to today", and "I felt isolated from the others today"). Items were answered on a fivepoint rating scale $(1=$ do not agree at all, $5=$ totally agree $)$. Daily loneliness was scored as the average of these items. Due to the data's nested structure, we computed nested alpha to estimate the scale's reliability (Nezlek, 2017). Reliability was .77 for Sample 1 and .77 for Sample 2.

\section{Statistical Analyses}

To conduct the analyses, we coded additional variables representing the date on which the survey was completed and whether the survey was completed on a weekend or a weekday. On the first day after implementing the pandemic-related measures (March 17, 2020), participants reported their daily loneliness for the first time. The variable days indicates the study's day, with 0 indicating the middle of the study period (i.e., the third Monday, March 30, 2020). The dummy variable for the weekdays vs. weekends was coded with 0 for all weekdays and with 1 for the weekend. This variable is referred to as weekend dummy. An example of this coding procedure is presented in the SM. 
We used multilevel models to predict daily loneliness over time. Daily loneliness (Level 1) was nested within persons (at Level 2). We present a sample model equation in the SM. All analyses were performed in R 3.6.2 (R Core Team, 2019). For the analyses, we used the R-packages Ime4 1.1.21 (Bates et al., 2015) and ImerTest 3.1.1 (Kuznetsova et al., 2017).

Our analytic procedure's aim was to find the best fitting model in Sample 1, which would then be replicated in Sample 2. We only interpreted effects that were significant $(p<.01)$ across both samples. All continuous Level 2 variables (i.e., age, number of household members, years of education) were grand-mean centered. Loneliness was z-standardized on the overall mean and standard deviation. Whenever we extended a model with additional predictors, we tested the model fit improvement using chi-square difference tests $(p<.01)$. We only continued extending the model if (a) the extended model converged and (b) model fit improved significantly.

First, we estimated a random intercept model in which daily loneliness was predicted by the linear time term days and the weekend dummy (see above for how these variables were defined). This model was then extended by a quadratic time term $\left(\right.$ days $\left.^{2}\right)$ and later by a cubic time term $\left(\right.$ days $\left.^{3}\right)$. After establishing the set of Level 1 predictors, we included all Level 2 covariates as additional predictors simultaneously (see Table S3).

Next, we estimated random slopes for all Level 1 predictors. We included random slopes for all Level 1 predictors simultaneously (i.e., for days, days ${ }^{2}$, days ${ }^{3}$, and weekend dummy). As this model did not converge, random slopes for days ${ }^{3}$ and days $^{2}$ were subsequently excluded until the model converged. In our final model, loneliness was predicted by days, days ${ }^{2}$, and weekend dummy, and random slopes were estimated for days and weekend dummy (see Table 1). We again included all Level 2 covariates as additional predictors simultaneously (see Table S4). 
In a final step, we added cross-level interactions for all Level 2 predictors separately, while controlling for fixed effects of all other Level 2 covariates. If a model did not converge, we reduced the number of random slopes until the model converged. Additionally, we estimated a model where we added cross-level interactions for all Level 2 predictors simultaneously (see Table S5).

\section{Results}

Daily loneliness increased in the first two weeks of the pandemic-related measures and decreased thereafter

The best fitting model identified in Sample 1 included the linear time term days, the quadratic time term days ${ }^{2}$, and the weekend dummy (Table 1). In both samples, we robustly found statistically significant non-linear changes in daily loneliness over time, with loneliness increasing by about 0.08 standard deviation $(S D)$ units during the first two weeks since implementing the pandemic-related measures, and then decreasing by about $0.14 S D$ units during the third and fourth weeks (Fig. 1B). Moreover, daily loneliness was lower on weekends (gray area in Fig. 1B) compared to weekdays.

Increases in daily loneliness over time were stronger for parents and increased with age

To test whether sociodemographic characteristics predicted changes in daily loneliness over time, we estimated separate multilevel models that included cross-level interactions between specific sociodemographic variables and linear time. In both samples, daily loneliness increased more strongly with increasing age $(\beta$ Sample $1=0.003, p<.001 ; \beta$ Sample $2=0.002, p=.002 ;$ Fig. $2 \mathrm{~A})$. Moreover, in both samples, changes in daily loneliness differed significantly according to parental status ( $\beta$ Sample 1 $=0.06, p=.001 ; \beta$ Sample $2=0.05, p=.005$; Fig. 2B). Over the four-week study period, daily loneliness decreased by about 0.05 SD units for people without children, but increased by about $0.13 \mathrm{SD}$ 
units for parents, controlling for all other sociodemographic characteristics. No other cross-level interactions between sociodemographic characteristics and linear time were robustly found in both samples (see Table S5).

\section{Discussion}

Our study describes changes in daily loneliness in a German sample during the first four weeks since implementing the pandemic-related measures. As an initial psychological reaction, average daily loneliness slightly increased during the first two weeks of the pandemic-related measures (from about March 17, 2020, to March 29, 2020). After that (from about March 30, 2020, to April 12, 2020), daily loneliness slightly decreased. Moreover, daily loneliness was lower on weekends compared to weekdays. Individuals greatly differed in their loneliness trajectories over time, as indicated by an increased model fit when including random slopes.

We identified sociodemographic subgroups who might be particularly vulnerable to changes in daily loneliness during the first four weeks since implementing the pandemic-related measures. Parents reported stronger increases in loneliness than people without children when controlling for other sociodemographic characteristics such as age, gender, and relationship status. During the first weeks since the implementation of the pandemic-related measures, parents were particularly challenged as they had to balance their job, childcare, other family activities, and, if necessary, homeschooling (Kreyenfeld et al., 2020). Consequently, they likely had less time to attend to their nonfamily social relationships during this phase, which may have led to an increase in loneliness. These explanations are, however, speculative, and further research is needed.

Moreover, we found that higher age was generally associated with lower average levels of loneliness. However, changes in daily loneliness were positively correlated with age, meaning that the 
older participants were, the more likely they were to report increasing levels of loneliness over time. Future research may examine whether modern technologies may have been more beneficial to younger people than to older people in buffering the effects of physical distancing policies. Single and widowed people and people with an increased health risk in the context of a Covid-19 infection reported higher daily loneliness. However, they did not report significantly stronger increases over time compared to those in a romantic relationship or to those with lower health risk.

Our results do not support the notion that loneliness levels have linearly increased during the first weeks after the implementation of pandemic-related measures. However, many people may have been able to buffer the effects of the Covid-19 pandemic with financial or social resources in the first few weeks, but these resources may be depleted more quickly for some groups than others (e.g., financial reserves may last less long for low-income people). Consequently, the long-term effects might differ from short-term changes as stressors accumulate over time, and some groups may suffer more than others. Note that the sample used in this study is not a nationally representative random sample, limiting the generalizability of the results. For example, as this study was conducted online, our sample may include a non-representative (e.g., relatively tech-savvy) proportion of adults aged 60+ years (see SM for more details).

To conclude, we identified subgroups that are at a higher risk for changes in daily loneliness during the first four weeks since the implementation of pandemic-related measures. However, there may be other vulnerable groups that we could not identify in the current work, and for whom the adverse effects of physical distancing measures could be more damaging. 


\section{References}

Arslan, R. C., Walther, M. P., \& Tata, C. S. (2020). formr: A study framework allowing for automated feedback generation and complex longitudinal experience-sampling studies using R. Behavior Research Methods, 52(I), 376-387. https://doi.org/ I 0.3758/s I 3428-01 9-0I 236-y

Bates, D., Maechler, M., Bolker, B., \& Walker, S. (20I5). Fitting linear mixed-effects models using Ime4. Journal of Statistical Software, 67(I), I-48. https://doi.org//0.18637/jss.v067.i0 I

Dehning, J., Zierenberg, J., Spitzner, F. P., Wibral, M., Neto, J. P., Wilczek, M., \& Priesemann, V. (2020). Inferring change points in the spread of COVID-19 reveals the effectiveness of interventions. Science, eabb9789. https://doi.org// 0.I I 26/science.abb9789

Elmer, T., Mepham, K., \& Stadtfeld, C. (2020). Students under lockdown: Assessing change in students' social networks and mental health during the COVID-I 9 crisis. https://psyarxiv.com/ua6tq/

English, T., \& Carstensen, L. L. (20I4). Selective narrowing of social networks across adulthood is associated with improved emotional experience in daily life. International Journal of Behavioral Development, 38(2), 195-202. https://doi.org/I0.1 I77/01650254I35I5404

Entringer, T., Kröger, H., Schupp, J., Kühne, S., Liebig, S., Goebel, J., Grabka, M. M., Graeber, D., Kroh, M., Schröder, C., Seebauer, J., \& Zinn, S. (2020). Psychische Krise durch Covid-19? Sorgen sinken, Einsamkeit steigt, Lebenszufriedenheit bleibt stabil. https://www.diw.de/documents/publikationen/73/diw_0I.c.79|307.de/diw_spl087.pdf

Fancourt, D., \& Steptoe, A. (2020). COVID-19 social study. https://www.nuffieldfoundation.org/project/covid-19-social-study

Faraway, J. J. (2016). Does data splitting improve prediction? Statistics and Computing, 26(I-2), 49-60. https://doi.org/| 0.1007/s | | 222-0|4-9522-9 
Finley, D. (2020, March 16). How increased social distancing for the coronavirus could spur a loneliness epidemic. BUSINESS INSIDER. https://www.businessinsider.com/coronavirus-could-spurloneliness-epidemic-2020-3?r=DE\&IR=T

Folk, D., Okabe-Miyamoto, K., Dunn, E., \& Lyubomirsky, S. (2020). Have introverts or extraverts declined in social connection during the first wave of COVID-/9? https://psyarxiv.com/tkr2b/

Fujiwara, D., Dolan, P., Lawton, R., Behzadnejad, F., Lagarde, A., Maxwell, C., \& Peytrignet, S. (2020). The Wellbeing Costs of COVID-19 in the UK. https://www.jacobs.com/sites/default/files/202005/jacobs-wellbeing-costs-of-covid-19-uk.pdf

Hawkley, L. C., Buecker, S., Kaiser, T., \& Luhmann, M. (2020). Loneliness from young adulthood to old age: Explaining age differences in loneliness in the United States. Unpublished Manuscript.

Holmes, E. A., O’Connor, R. C., Perry, V. H., Tracey, I., Wessely, S., Arseneault, L., Ballard, C., Christensen, H., Silver, R. C., Everall, I., Ford, T., John, A., Kabir, T., King, K., Madan, I., Michie, S., Przybylski, A. K., Shafran, R., Sweeney, A., ... Bullmore, E. (2020). Multidisciplinary research priorities for the COVID-19 pandemic: A call for action for mental health science. The Lancet Psychiatry, 7(6), 547-560. https://doi.org/I0.1016/S22I 5-0366(20)30I68-I

Holt-Lunstad, J., Smith, T. B., Baker, M., Harris, T., \& Stephenson, D. (2015). Loneliness and social isolation as risk factors for mortality: A meta-analytic review. Perspectives on Psychological Science, I0(2), 227-237. https://doi.org//0.1 I77//74569/6/4568352

Hutteman, R., Hennecke, M., Orth, U., Reitz, A. K., \& Specht, J. (20I4). Developmental tasks as a framework to study personality development in adulthood and old age. European Journal of Personality, 28(3), 267-278. https://doi.org/10.1002/per.1959 
Klein, E. (2020, March 12). Coronavirus will also cause a loneliness epidemic. Vox.

https://www.vox.com/2020/3/I 2/2 I I73938/coronavirus-covid-19-social-distancing-elderly-epidemic-isolation-quarantine

Kreyenfeld, M., Zinn, S., Entringer, T., Goebel, J., Grabka, M. M., Graeber, D., Kroh, M., Kröger, H., Kühne, S., Liebig, S., Schröder, C., Schupp, J., \& Seebauer, J. (2020). Coronavirus \& care: How the coronavirus crisis affected fathers' involvement in Germany. SOEPpapers on Multidisciplinary Panel Data Research. https://www.diw.de/documents/publikationen/73/diw_0l.c.794183.de/diw_spl096.pdf

Kuznetsova, A., Brockhoff, P. B., \& Christensen, R. H. B. (2017). ImerTest package: Tests in linear mixed effects models. Journal of Statistical Software, 82(I3), I-26. https://doi.org//0.18637/jss.v082.il 3

Luchetti, M., Lee, J. H., Aschwanden, D., Sesker, A., Strickhouser, J. E., Terracciano, A., \& Sutin, A. R. (2020). The trajectory of loneliness in response to COVID-19. American Psychologist. https://doi.org/10.1037/amp0000690

Luhmann, M., \& Hawkley, L. C. (2016). Age differences in loneliness from late adolescence to oldest old age. Developmental Psychology, 52(6), 943-959. https://doi.org//0.1037/dev0000I I 7

Mihalopoulos, C., Le, L. K.-D., Chatterton, M. L., Bucholc, J., Holt-Lunstad, J., Lim, M. H., \& Engel, L. (2020). The economic costs of loneliness: A review of cost-of-illness and economic evaluation studies. Social Psychiatry and Psychiatric Epidemiology, 55(7), 823-836. https://doi.org/10.1007/s00127-019-01733-7

Nezlek, J. B. (2017). A practical guide to understanding reliability in studies of within-person variability. Journal of Research in Personality, 69, I49-I55. https://doi.org/I0.1016/j.jrp.2016.06.020 
Peplau, L. A., \& Perlman, D. (1982). Loneliness: A sourcebook of current theory, research and therapy. Wiley.

Pinquart, M., \& Sörensen, S. (2003). Risk factors for loneliness in adulthood and old age: A meta-analysis. In S. P. Shohov (Ed.), Advances in psychology research (Vol. 19, pp. II I-I43). Nova Science Publishers.

R Core Team. (2019). R: A language and environment for statistical computing. $R$ Foundation for Statistical Computing, Vienna, Austria. https://www.r-project.org/

Sweeny, K., Rankin, K., Cheng, X., Hou, L., Long, F., Yao Meng, Lilian Azer, Renlai Zhou, \& Weiwei Zhang. (2020). Flow in the Time of COVID-19: Findings from China. https://psyarxiv.com/e3kcw/

Weston, S. J., Ritchie, S. J., Rohrer, J. M., \& Przybylski, A. K. (2019). Recommendations for increasing the transparency of analysis of preexisting data sets. Advances in Methods and Practices in Psychological Science, 2(3), 214-227. https://doi.org/I0.II77/25/52459/9848684

Yarkoni, T., \& Westfall, J. (2017). Choosing prediction over explanation in psychology: Lessons from machine learning. Perspectives on Psychological Science, I2(6), I I00-1 I 22.

https://doi.org//0.1177/174569/617693393 
Tables and Figures

Table I. Fixed effect estimates from random slopes models for loneliness in both samples.

\begin{tabular}{|c|c|c|c|c|c|c|}
\hline \multirow[b]{2}{*}{ Variables } & \multicolumn{3}{|l|}{ Sample I } & \multicolumn{3}{|l|}{ Sample 2} \\
\hline & Estimate & $S E$ & $p$ & Estimate & $S E$ & $p$ \\
\hline \multicolumn{7}{|l|}{ Model I } \\
\hline Intercept & 0.009 & 0.019 & .644 & 0.001 & 0.020 & .957 \\
\hline Days $^{\mathrm{a}}$ & -0.006 & 0.008 & .452 & -0.004 & 0.008 & .625 \\
\hline Days $^{2}$ & -0.026 & 0.006 & $<.001$ & -0.024 & 0.006 & $<.001$ \\
\hline WD: weekdayb & 0.037 & 0.012 & .003 & 0.049 & 0.013 & $<.001$ \\
\hline
\end{tabular}

Notes. SE = Standard Error; aThe variable days indicates the date of the assessment and is centered such that 0 represents the beginning of the third week (March 30, 2020).

A

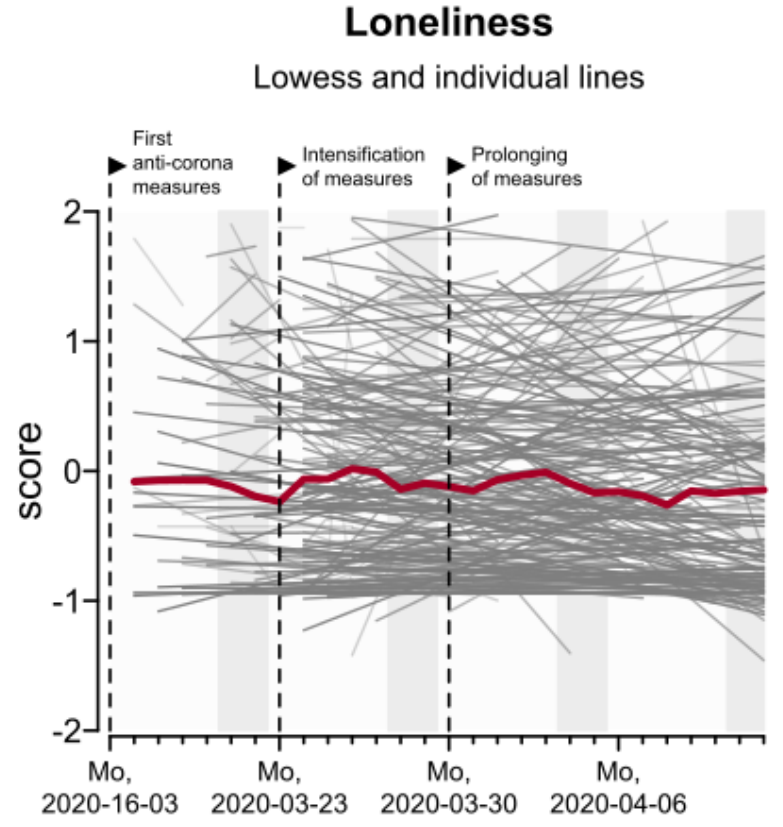

B

\section{Loneliness}

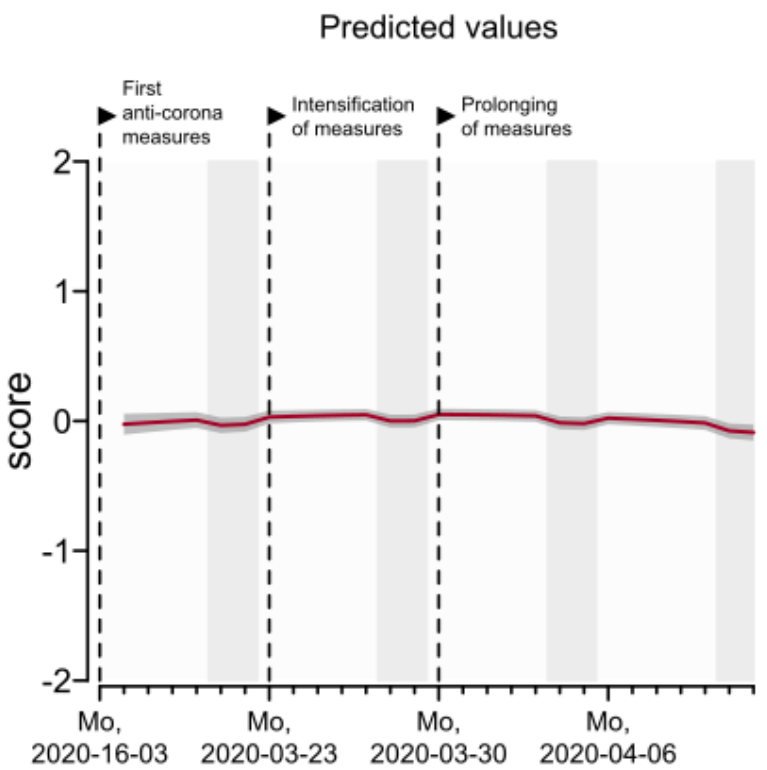

Fig. I.

Changes in daily loneliness throughout the study. (A) Grey lines represent individual predicted levels of daily loneliness (z-standardized) for 250 randomly selected participants from Sample 2, the red (bold) line represents a LOWESS line fitted to Sample 2, (B) the red (bold) line represents predicted values from the final random coefficient model in Sample 2, flanked by $99 \%$ confidence intervals. Gray columns indicate weekends. 
Fig. 2.

A

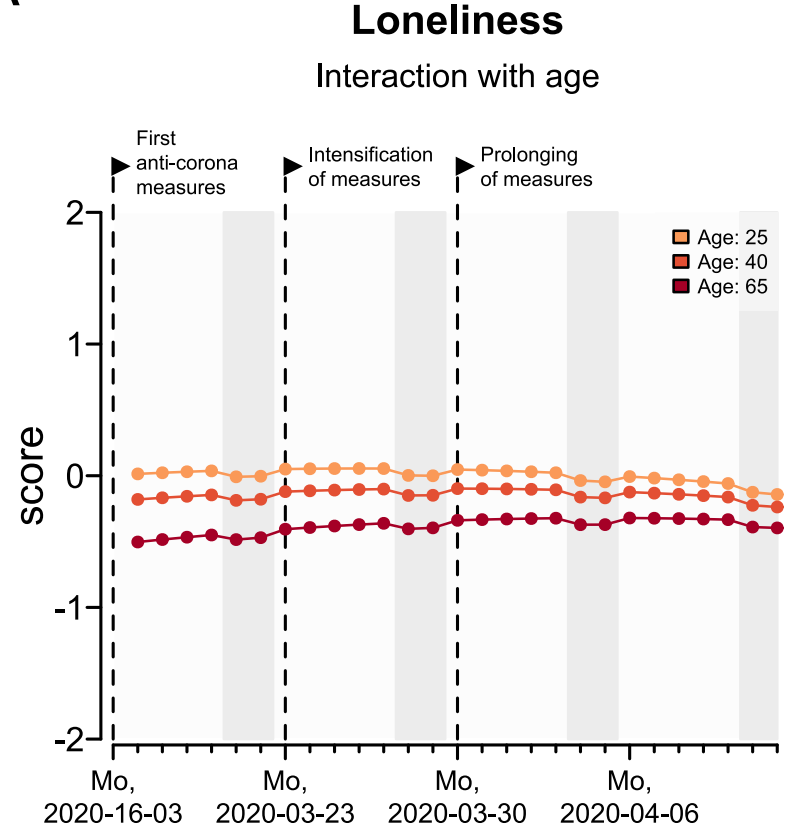

B

Loneliness

Interaction with parental status

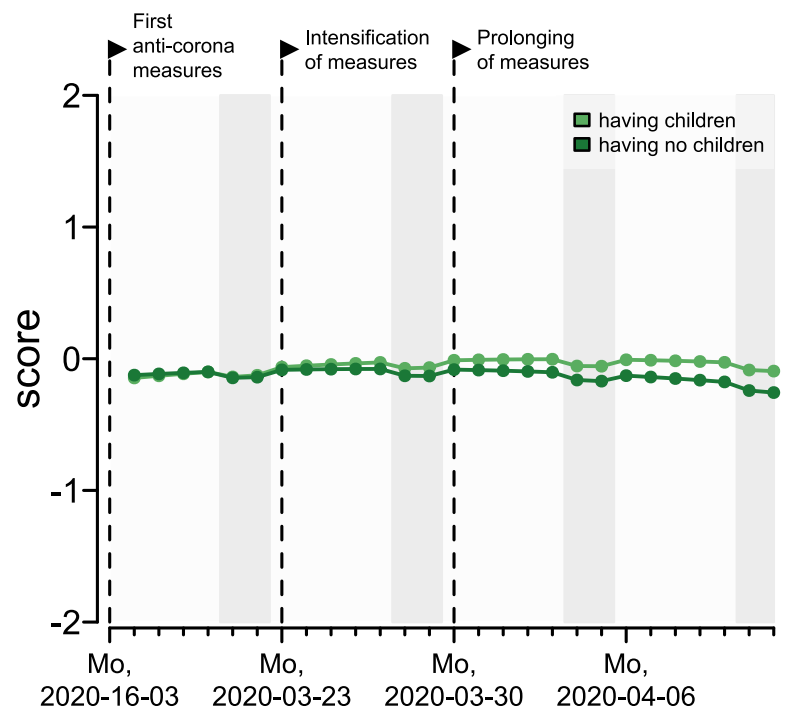

Age and parental status predict changes in daily loneliness over time. (A) Predicted levels of daily loneliness (z-standardized) for different ages from Sample 2. (B) predicted levels of daily loneliness for different levels of parental status from Sample 2. Gray columns indicate weekends. In both models, we controlled for gender, years of education, employment status, occupational classification, relationship status, number of household members, parental status, and pre-existing health risk condition. 


\section{Acknowledgments}

We thank our persistent study participants who supported this ongoing line of research. Moreover, we thank our research assistants and interns (Anke Kirchdörfer, Melina Sostmann, Vivien Janowicz, Malin Wigger, and Sandy Chwastek) for the recruitment and support of our study participants. The data for this study were collected with the survey software formr. We thank the entire formr team (Ruben C. Arslan, Cyril S. Tata, and Matthias P. Walther) for their technical assistance during data collection and for providing the formr platform.

\section{Author contributions}

Susanne Buecker: Conceptualization, Methodology, Formal Analysis, Investigation, Resources, Writing - Original Draft, Writing - Review \& Editing, Project Administration; Kai T. Horstmann: Methodology, Investigation, Resources, Software, Data Curation, Writing - Original Draft, Writing - Review \& Editing, Visualization; Julia Krasko: Investigation, Resources, Writing - Review \& Editing, Visualization; Sarah Kritzler: Investigation, Resources, Writing - Review \& Editing, Visualization; Sophia Terwiel: Investigation, Resources, Writing - Review \& Editing, Data Curation; Till Kaiser: Data Curation, Writing - Review \& Editing; Maike Luhmann: Conceptualization, Methodology, Writing - Original Draft, Writing - Review \& Editing, Supervision.

\section{Declaration of Conflicting Interests}

The author(s) declared no potential conflicts of interest with respect to the research, authorship, and/or publication of this article.

\section{Funding}

No specific funding was used.

\section{Further Materials}

Supplementary Material: https://osf.io/wqc25/ 